\title{
Identification of physical nonlinearities of a hybrid aeroelastic-pressure balance
}

\author{
Zengshun Chen $\cdot$ K. T. Tse $i$
}

Received: 19 June 2017 / Accepted: 29 July 2019 / Published online: 13 August 2019

(C) The Author(s) 2019

\begin{abstract}
This study has presented an improved method for determining physical nonlinearities of weakly nonlinear spring-suspension system and successfully applied to a novel hybrid aeroelastic-pressure balance (HAPB) system used in wind tunnel, which can be used for simultaneously obtaining the unsteady wind pressure and aeroelastic response of a test model. A nonlinear identification method of equivalent linearization approximation was firstly developed on the basis of the averaging method of Krylov-Bogoliubov to model the physical nonlinearity of a weakly nonlinear system. Subsequently, the nonlinear physical frequency and damping were identified using a modified Morlet wavelet transform method and a constant variant method. Using these methods, the physical nonlinear frequency and damping of the HAPB system with a vertical test model were determined and were validated by a time domain method and the Newmark- $\beta$ method. Finally, the nonlinear mechanical frequency and damping of the HAPB system with inclined test models
\end{abstract}

\section{Z. Chen}

School of Civil Engineering, Chongqing University, Chongqing 400033, China

e-mail: zchenba@connect.ust.hk

K. T. Tse ( $\varangle)$

Department of Civil and Environmental Engineering, The Hong Kong University of Science and Technology, Clear Water Bay, Kowloon, Hong Kong, China

e-mail: timkttse@ust.hk

\section{Z. Chen}

NatHaz Modeling Laboratory, University of Notre Dame, Notre Dame, IN 46556, USA were determined in a similar way. This study has not only provided an identification method for determining physical nonlinearities of weakly nonlinear system, but presented the detail for developing a hybrid aeroelastic-pressure balance used in wind tunnel.

Keywords Physical nonlinearities - Equivalent linearization approximation - Modified Morlet wavelet transform $\cdot$ Hybrid aeroelastic-pressure balance

\section{Introduction}

Conventionally, a linear mechanical vibration model is utilized to determine the physical nonlinearities (nonlinear damping and stiffness) of a system from a free decay test in wind and offshore engineering [14]. Using the values identified by the linear model, responses of various engineering objects are predicted. However, as pointed out by Staszewski [5], all physical and engineering systems inevitably exhibit in practice nonlinear behaviors which may arise from structural, geometric and material properties. A linear model applied to a nonlinear system would result in significant inaccuracies in response predictions of the system. Though the nonlinear frequency and damping of a weakly nonlinear system may vary slowly with oscillating amplitude, and the physical nonlinearities of the system are small in quantity, their presence would lead to different dynamic behaviors, such as the longduration behaviors of energy dissipation and phase 
modulation due to the time-varying nonlinearities of the system. Since a nonlinear system can display complex phenomenon that a linear system cannot, the distinction between a linear and a nonlinear system should be well concerned.

The most common method to identify the physical nonlinearities of a system is the Hilbert transform (HT) method, which was developed in 1980s [6]. It utilizes the transit amplitude and frequency of the impulse response function to obtain the backbone curve and quantitative information about the nonlinear behavior of the system [5]. Due to its convenience, the method has received much attention and been widely utilized to many systems [7-9]. Despite its success, it has some limitations. For example, it is valid only for asymptotic signals and the 'end effect' or 'Gibbs effect' may be significant due to the incomplete data periodicity in the discrete HT $[10,11]$. Combining the HT method and an empirical mode decomposition method, the HilbertHuang transform (HHT) method was proposed and has been utilized to analyze nonlinear and nonstationary time series [12]. The HHT method is effective in signal decomposition and time-frequency domain analysis, but it could not separate closely spaced multi-frequency signals into a set of mono-frequency components. The Wigner-Ville distribution [13], Gabor transform [14] and Wavelet transform [15-17] have also been developed and provided the most effective results for singledegree-of-freedom (SDOF) systems. However, they have been proved to be difficult for nonlinearities of multi-degree-of-freedom (MDOF) systems since the analysis requires bandpass filtration of the signal. To avoid the problems, a few attempts have been made to identify nonlinear parameters of MDOF systems, i.e., the improved HHT [18], the improved Wavelet transform [5,15], and the harmonic balance method [19]. For weakly nonlinear systems, other methods have also been developed including the equivalent linearization method [20], the force-state mapping method [21], and the restoring force surface method [22], etc. These methods are complicated in use, and the accuracy is not good enough for weakly nonlinear systems.

A high-frequency base balance (HFBB) or synchronous multi-pressure sensing system (SMPSS) test technique is often utilized to obtain aerodynamic performances of bluff bodies, such as building and bridge models, and an aeroelastic test technique is frequently carried out for the evaluation of aeroelastic performances $[23,24]$. However, both the HFBB and SMPSS techniques are static force measurements, which means that the techniques cannot consider the effect of structural motion that may have great effect on the evaluation of aerodynamic and aeroelastic performances of a structure. The aeroelastic test is used for obtaining aeroelastic response, but it cannot give aerodynamic forces on a test model simultaneously. To comprehensively study the effect of structural motion, both the aerodynamic force and aeroelastic response measurements of a structure are required. To the author's best knowledge, very few studies have concentrated on the hybrid aeroelastic-pressure balance used in wind tunnel.

This study aims to (1) propose a method to identify the physical nonlinearity of weakly nonlinear systems; (2) to devise a novel hybrid aeroelastic-pressure balance (HAPB) test system and identify the physical nonlinearities of the system using the proposed method. In Sect. 2, the solution of a weak nonlinear system is derived from the averaging method of KrylovBogoliubov and the ELA method. Subsequently, a modified Morlet wavelet transform (MMWT) method is proposed and utilized for the identification of the nonlinearities of a weakly nonlinear system. A constant variant method is also employed to determine the nonlinear damping of the system. In Sect. 3, a HAPB system is devised and the physical damping and stiffness of the system were identified by a conventional linear model. The physical damping and stiffness identified by the linear model are constant, and the linear model has been proved to be not precise enough in the prediction of long-term free decay response due to the ignorance of the slow varying characteristics of the system. In Sect. 4, the physical nonlinearities of the HAPB system with a vertical test model are determined by using the methods developed in Sect. 2. The nonlinearities are validated by comparing the obtained nonlinearities with those obtained from a time domain method and are further verified by comparing the response calculated from the identified nonlinearities with the response directly observed from a free decay test. After verifying the nonlinearities of the HAPB system with a vertical test model, Sect. 5 presents the physical nonlinearities of the system with inclined test models. The present study makes sense in several aspects: (1) the analytical methods can be used for identifying physical nonlinearities of weakly nonlinear systems; (2) a HAPB system that can measure the aeroelastic and aerodynamic performance of structures was devised; (3) the identified 
nonlinearities of the HAPB system are potential to be employed to the dynamic analysis of a test system, such as the analysis of the wind-induced oscillation of bluff bodies (e.g., building and bridge pylon or deck models).

\section{Nonlinear identification model}

\subsection{Equivalent linearization approximation}

In an equivalent linearization approximation (ELA), the response of a weakly nonlinear system (SDOF) is regarded as a perturbation of undamped oscillator, and the general differential equation governing the free decay response of the system is expressed as

$\ddot{u}(t)+\varepsilon f[\dot{u}(t), u(t)]+\omega_{0}^{2} u=0$

where $\varepsilon$ is a small dimensionless parameter and $0<$ $\varepsilon<<1 . f(\dot{u}, u)$ is a general nonlinear function of displacement $u$ and velocity $\dot{u}$. It is well known that the solution of Eq. (1), when $\varepsilon=0$ (linear problem), is $u(t)=A \cos \left(\omega_{0} t+\varphi\right)$ where $A$ and $\varphi$ are constants. When $\varepsilon \neq 0$, the solution of Eq. (1) can be determined by the averaging method of Krylov-Bogoliubov $[15,25]$, and is expressed as

$u(t)=A(t) \cos \left[\omega_{0} t+\varphi(t)\right]$

where $A(t)$ and $\varphi(t)$ are the amplitude and phase modulation of the free decay response of a nonlinear vibrating system and they are time-dependent functions. Then, the first-order derivatives of Eq. (2) is expressed as

$$
\begin{aligned}
\dot{u}(t)= & \dot{A}(t) \cos \left[\omega_{0} t+\varphi(t)\right] \\
& -A(t)\left[\omega_{0}+\dot{\varphi}(t)\right] \sin \left[\omega_{0} t+\varphi(t)\right]
\end{aligned}
$$

Suppose the velocity of the free decay response has the same form as the harmonic oscillator and is expressed as

$$
\dot{u}(t)=-A(t) \omega_{0} \sin \left[\omega_{0} t+\varphi(t)\right]
$$

The second-order derivative of the harmonic oscillator is determined based on Eq. (4).

$$
\begin{aligned}
\ddot{u}(t)= & -\dot{A}(t) \omega_{0} \sin \left[\omega_{0} t+\varphi(t)\right] \\
& -A(t) \omega_{0}\left[\omega_{0}+\dot{\varphi}(t)\right] \cos \left[\omega_{0} t+\varphi(t)\right]
\end{aligned}
$$

Comparing Eq. (3) with Eqs. (4), (6) is determined as

$$
\begin{aligned}
& \dot{A}(t) \omega_{0} \cos \left[\omega_{0} t+\varphi(t)\right] \\
& \quad-A(t) \dot{\varphi}(t) \sin \left[\omega_{0} t+\varphi(t)\right]=0
\end{aligned}
$$

Introducing all the relations in Eqs. (4)-(6) into the differential equation Eqs. (1), (7) is obtained as

$$
\begin{aligned}
& \dot{A}(t) \omega_{0} \sin [\psi(t)]+A(t) \omega_{0} \dot{\varphi}(t) \cos [\psi(t)] \\
& \quad=-\varepsilon f\left\{A(t) \cos [\psi(t)],-A(t) \omega_{0} \sin [\psi(t)]\right\}
\end{aligned}
$$

where $\psi(t)=\omega_{0} t+\varphi(t)$.

Solving Eqs. (6) and (7), $\dot{A}(t)$ and $\dot{\varphi}(t)$ are obtained as

$$
\begin{aligned}
\dot{A}(t)= & \frac{\varepsilon}{\omega_{0}} \sin [\psi(t)] f\{A(t) \cos [\psi(t)], \\
& \left.-A(t) \omega_{0} \sin [\psi(t)]\right\} \\
\dot{\varphi}(t)= & \frac{\varepsilon}{\omega_{0} A(t)} \cos [\psi(t)] f\{A(t) \cos [\psi(t)], \\
& \left.-A(t) \omega_{0} \sin [\psi(t)]\right\}
\end{aligned}
$$

Applying Fourier expansion to Eqs. (8) and (9), we have

$$
\begin{aligned}
f & \left\{A(t) \cos [\psi(t)],-A(t) \omega_{0} \sin [\psi(t)]\right\} \sin [\psi(t)] \\
& =K_{0}(A)+\sum_{n=1}^{\infty}\left[K_{n}(A) \cos n \psi+L_{n}(A) \sin n \psi\right]
\end{aligned}
$$

$$
\begin{aligned}
f & \left\{A(t) \cos [\psi(t)],-A(t) \omega_{0} \sin [\psi(t)]\right\} \cos [\psi(t)] \\
& =P_{0}(A)+\sum_{n=1}^{\infty}\left[P_{n}(A) \cos n \psi+Q_{n}(A) \sin n \psi\right]
\end{aligned}
$$

where

$$
\begin{aligned}
K_{0}(A)= & \frac{1}{2 \pi} \int_{0}^{2 \pi} f\{A(t) \cos [\psi(t)], \\
& \left.-A(t) \omega_{0} \sin [\psi(t)]\right\} \sin [\psi(t)] \mathrm{d} \psi \\
P_{0}(A)= & \frac{1}{2 \pi} \int_{0}^{2 \pi} f\{A(t) \cos [\psi(t)], \\
& \left.-A(t) \omega_{0} \sin [\psi(t)]\right\} \cos [\psi(t)] \mathrm{d} \psi \\
K_{n}(A)= & \frac{1}{\pi} \int_{0}^{2 \pi} f\{A(t) \cos [\psi(t)], \\
& \left.-A(t) \omega_{0} \sin [\psi(t)]\right\} \sin [\psi(t)] \cos [n \psi(t)] \mathrm{d} \psi \\
P_{n}(A)= & \frac{1}{\pi} \int_{0}^{2 \pi} f\{A(t) \cos [\psi(t)], \\
& \left.-A(t) \omega_{0} \sin [\psi(t)]\right\} \cos [\psi(t)] \cos [n \psi(t)] \mathrm{d} \psi \\
L_{n}(A)= & \frac{1}{\pi} \int_{0}^{2 \pi} f\{A(t) \cos [\psi(t)], \\
& \left.-A(t) \omega_{0} \sin [\psi(t)]\right\} \sin [\psi(t)] \sin [n \psi(t)] \mathrm{d} \psi \\
Q_{n}(A)= & \frac{1}{\pi} \int_{0}^{2 \pi} f\{A(t) \cos [\psi(t)], \\
& \left.-A(t) \omega_{0} \sin [\psi(t)]\right\} \cos [\psi(t)] \sin [n \psi(t)] \mathrm{d} \psi
\end{aligned}
$$


Then, Eqs. (8) and (9) can be rewritten by

$$
\begin{aligned}
\dot{A}(t)= & -\frac{\varepsilon}{\omega_{0}} K_{0}(A) \\
& -\frac{\varepsilon}{\omega_{0}} \sum_{n=1}^{\infty}\left[K_{n}(A) \cos n \psi+L_{n}(A) \sin n \psi\right] \\
\dot{\varphi}(t)= & -\frac{\varepsilon}{\omega_{0} A(t)} P_{0}(A) \\
& -\frac{\varepsilon}{\omega_{0} A(t)} \sum_{n=1}^{\infty}\left[P_{n}(A) \cos n \psi+Q_{n}(A) \sin n \psi\right]
\end{aligned}
$$

For a weakly nonlinear system, the variation of $\dot{A}(t)$ and $\dot{\varphi}(t)$ is small because of the small parameter $\varepsilon$, so that the variation could be approximated by their changes in the corresponding single period. Therefore, based on the method of Krylov-Bogoliubov, $\dot{A}(t)$ and $\dot{\varphi}(t)$ can be approximated by the zero-harmonic term of Fourier series and are expressed as

$$
\begin{aligned}
\dot{A}(t)= & -\frac{\varepsilon}{\omega_{0}} K_{0}(A) \\
= & -\frac{\varepsilon}{2 \pi \omega_{0}} \int_{0}^{2 \pi} f\{A(t) \cos [\psi(t)], \\
& \left.-A(t) \omega_{0} \sin [\psi(t)]\right\} \sin [\psi(t)] \mathrm{d} \psi \\
\dot{\varphi}(t)= & -\frac{\varepsilon}{\omega_{0} A(t)} P_{0}(A) \\
= & -\frac{\varepsilon}{2 \pi \omega_{0} A(t)} \int_{0}^{2 \pi} f\{A(t) \cos [\psi(t)], \\
& \left.-A(t) \omega_{0} \sin [\psi(t)]\right\} \cos [\psi(t)] \mathrm{d} \psi
\end{aligned}
$$

Equations (20) and (21) allow to readily obtain an approximate analytical solution describing the oscillating behavior of a weakly nonlinear system, for different forms of the nonlinear function $f(\dot{u}, u)$. The solution of Eq. (1) using the method of Krylov-Bogoliubov is the same with that using the method of multiple scales introduced in previous studies [10,26-28].

An equivalent linearization approximation method is then applied to model the physical nonlinearities of a weakly nonlinear system by introducing a damping coefficient $D(A)$ which is in-phase with oscillation velocity and a restoring force coefficient $S(A)$ which is in-phase with oscillation displacement, defined in Eqs. (22) and (23).

$$
\begin{aligned}
D(A)= & \frac{\varepsilon}{\pi A(t) \omega_{0}} \cdot \int_{0}^{2 \pi} f\{A(t) \cos [\psi(t)], \\
& \left.-A(t) \omega_{0} \sin [\psi(\mathrm{t})]\right\} \sin [\psi(t)] \mathrm{d} \psi \\
K(A)= & \omega_{0}^{2}+\frac{\varepsilon}{\pi A(t)} \cdot \int_{0}^{2 \pi} f\{A(t) \cos [\psi(t)], \\
& \left.-A(t) \omega_{0} \sin [\psi(t)]\right\} \cos \psi(t) \mathrm{d} \psi
\end{aligned}
$$

Substituting Eqs. (20) and (21) into Eqs. (22) and (23), respectively, we have

$\dot{A}=\frac{D(A)}{2} \cdot A$

$\dot{\varphi}=\omega_{\mathrm{e}}(A)-\omega_{0}$

where $\omega_{\mathrm{e}}(A)=\sqrt{K(A)}$.

Differentiating Eq. (2) with respect to $t$ and combining it with Eqs. (24) and (25), we have

$$
\begin{aligned}
\dot{u}(t)= & \dot{A}(t) \cdot \cos \left[\omega_{0} t+\varphi(t)\right] \\
& -A(t) \cdot \sin \left[\omega_{0} t+\varphi(t)\right] \cdot\left[\omega_{0}+\dot{\varphi}(t)\right] \\
= & \frac{D(A)}{2} \cdot u-A \cdot \omega_{\mathrm{e}}(A) \cdot \sin \psi \\
\ddot{u}(t)= & \frac{1}{2} \frac{\mathrm{d} D(A)}{\mathrm{d} A} \cdot \frac{\mathrm{d} A}{\mathrm{~d} t} \cdot u \\
& +\frac{D(A)}{2} \cdot\left[\frac{D(A)}{2} \cdot u-A \cdot \omega_{\mathrm{e}}(A) \cdot \sin \psi\right] \\
& -\dot{A} \cdot \omega_{\mathrm{e}}(A) \cdot \sin \psi \\
& -A \cdot\left[\frac{\mathrm{d} \omega_{\mathrm{e}}(A)}{\mathrm{d} A} \cdot \frac{\mathrm{d} A}{\mathrm{~d} t} \cdot \sin \psi\right. \\
& \left.+\omega_{\mathrm{e}}(A) \cdot \cos \psi \cdot\left(\omega_{0}+\dot{\varphi}\right)\right] \\
= & -D(A) \dot{u}-K(A) u+\frac{D^{2}(A)}{4} u \\
& -\frac{1}{4} \frac{\mathrm{d} D(A)}{\mathrm{d} A} D(A) \cdot A \cdot u \\
& -\frac{\mathrm{d} \omega_{\mathrm{e}}(A)}{\mathrm{d} A} \cdot \frac{D(A)}{2} \cdot A^{2} \cdot \sin \psi
\end{aligned}
$$

Rewriting Eq. (27), we have

$$
\begin{aligned}
\ddot{u}(t) & +D(A) \dot{u}+K(A) u \\
= & \frac{D^{2}(A)}{4} u-\frac{1}{4} \frac{\mathrm{d} D(A)}{\mathrm{d} A} D(A) \cdot A \cdot u \\
& -\frac{\mathrm{d} \omega_{\mathrm{e}}(A)}{\mathrm{d} A} \cdot \frac{D(A)}{2} \cdot A^{2} \cdot \sin \psi
\end{aligned}
$$


From Eqs. (24) and (25), we know

$$
\begin{aligned}
D(A) & =o(\varepsilon) \\
\frac{\mathrm{d} D(A)}{\mathrm{d} A} & =\frac{2}{A^{2}}\left(A \cdot \frac{\mathrm{d} A^{\prime}}{\mathrm{d} A}-A^{\prime}\right)=o(\varepsilon) \\
\frac{\mathrm{d} \omega_{\mathrm{e}}(A)}{\mathrm{d} A} & =\frac{\mathrm{d} \varphi^{\prime}(A)}{\mathrm{d} A}=o(\varepsilon)
\end{aligned}
$$

Combining Eq. (28) with Eqs. (29)-(31), we have

$\ddot{u}(t)+D(A) \dot{u}(t)+K(A) u(t)=o\left(\varepsilon^{2}\right)$

By using equivalent viscous damping and frequency, Eq. (32) can be further expressed as

$\ddot{u}(t)+2 \omega_{\mathrm{e}}(A) \xi_{\mathrm{e}}(A) \dot{u}+\omega_{\mathrm{e}}^{2}(A) u=o\left(\varepsilon^{2}\right)$

where $\xi_{\mathrm{e}}(A)$ and $\omega_{\mathrm{e}}(A)$ are equivalent amplitudedependent damping ratio and amplitude-dependent circular frequency, respectively.

From Eq. (33), the damping and frequency of a nonlinear system are approximated by a first-order approximation. Comparing Eq. (33) with the linear model of Eq. (1), the nonlinear damping and frequency of a weakly nonlinear system are amplitude-dependent and would be accurate to model the physical nonlinearities of a spring-suspension weakly nonlinear system.

\subsection{System identification}

To determine the slow varying amplitude-dependent damping and frequency in Eq. (33), a modified Morlet wavelet transform (MMWT) method and a constant variant method are proposed hereunder. These methods will be verified and applied to the identification of the nonlinearities of a weakly nonlinear system.

\subsubsection{Modified Morlet wavelet transform and wavelet entropy}

One can refer to the background of the continuous wavelet transform in previous studies $[29,30]$. Herein, a modified Morlet wavelet transform function with a bandwidth parameter $f_{\mathrm{b}}$ that controls the shape of the wavelet is directly defined as

$\lambda(t)=\frac{1}{\sqrt{\pi f_{\mathrm{b}}}} e^{-t^{2} / f_{\mathrm{b}}} e^{i \omega_{0} t}$

where $f_{\mathrm{b}}$ is a bandwidth parameter and can give a narrower bandwidth allowing a better frequency resolution, but at the expense of time resolution. Therefore, there exists an optimal $f_{\mathrm{b}}$ that balances the time and frequency resolution of a certain signal localized in the time-frequency domain. The modified MMWT function offers a better compromise in both time and frequency of a signal, than a traditional Morlet wavelet function. The optimal bandwidth parameter can be determined by minimizing the entropy of the wavelet coefficients introduced hereunder.

Assume that the signal $u(t)$ is given by a series of sampled values $\{u(n)\}$, where $n=1,2, \ldots N$. In the wavelet multi-resolution analysis of the time series $\{u(n)\}$, the energy for each scale $a_{i}$ is

$E_{a i}=\sum_{j}\left|W_{\lambda}[u]\left(a_{i}, b_{j}\right)\right|^{2}$

where $W_{\lambda}[u]\left(a_{i}, b_{j}\right)$ are a set of wavelet coefficients over a number of translations $b_{j}$. The total energy is then obtained by $E_{\text {total }}=\sum_{i} E_{a i}$, and the normalized energy is obtained by $E_{p i}=E_{a i} / E_{\text {total }}$ representing the relative energy for different $i$. The distribution of $E_{p i}$ is considered as a time-scale density and the Shannon entropy $[15,31]$ is a useful tool to analyze and compare the distribution. Based on the Shannon entropy, the time-varying wavelet entropy is defined as

$$
\mathrm{WE}=\sum_{i} E_{p i} \log \left(E_{p i}\right)
$$

From Eq. (42), the optimal bandwidth parameter $f_{\mathrm{b}}$ is determined by minimization of the wavelet entropy.

\subsubsection{The ridge and skeleton of the modified Morlet wavelet transform}

A class of signals called asymptotic was defined and some results for the time-frequency analysis of the signals were obtained in a previous study [15]. Based on the study, a signal in form of Eq. (2) is asymptotic if the amplitude $A(t)$ varies slowly compared to the variations of the phase $\varphi(t)$. From the definition, the signal is expressed as $u_{a}(t)=A(t) e^{i \varphi(t)}$ and the time-varying angular frequency is $\omega(t)=\dot{\varphi}(t)$. Then, the continuous wavelet transform of an asymptotic signal $u(t)$ is obtained by asymptotic techniques and is expressed as [15]

$W_{\lambda}[u](a, b)=\frac{\sqrt{a}}{2} A(b) e^{i \varphi(b)} \hat{\lambda}[a \dot{\varphi}(b)]$

where $\hat{\lambda}$ is the dilated version of the Fourier transform of Eq. (34) and $\hat{\lambda}(a \omega)=e^{-f_{\mathrm{b}} / 4\left(a \omega-\omega_{0}\right)^{2}}$. 
Using the MMWT defined in Eqs. (34), (37) is rewritten as

$W_{\lambda}[u](a, b)=\frac{\sqrt{a}}{2} A(b) e^{i \varphi(b)} e^{-f_{\mathrm{b}} / 4\left(a \dot{\varphi}(b)-\omega_{0}\right)^{2}}$

The term $e^{-f_{\mathrm{b}} / 4\left(a \dot{\varphi}(b)-\omega_{0}\right)^{2}}$ is interpreted as an energy density distribution over the time-scale plane. The concentration region of the energy on the time-scale plane is called the ridge of the continuous wavelet transform. The region corresponds to the maximum amplitude of the continuous wavelet transform. The ridges are identified by searching out the maximum local coefficients of the continuous wavelet transform: for each value of $b$, a value of $a$ is determined such as $\left|W_{\lambda}[u](a(b), b)\right|=\max _{a}\left|W_{\lambda}[u](a, b)\right|$. To obtain the ridge, the dilatation parameter, $a=a(b)=\omega_{0} / \dot{\varphi}(b)$, has to be determined by maximizing the $\hat{\lambda}[a \dot{\varphi}(b)]$ using the MMWT. We obtain

$W_{\lambda}[u](a(b), b)=\frac{\sqrt{a(b)}}{2} A(b) e^{i \varphi(b)}$

From Eq. (37), the real component of the MMWT along the ridge is directly proportional to the signal given by Eq. (2), and we have

$$
\begin{aligned}
& A(b)=2 \frac{W_{\lambda}[u](a(b), b)}{\sqrt{a(b)}} \\
& \varphi(b)=\operatorname{ang}\left(W_{\lambda}[u](a(b), b)\right)
\end{aligned}
$$

The ridge and skeleton will be used for the estimation of the instantaneous oscillating amplitude $A(t)$ and instantaneous angular frequency $\omega_{\mathrm{e}}(A)=\dot{\varphi}(t)$. It should be noted that since the identified circular frequency $\omega_{\mathrm{e}}(A)$ varies slowly with the variable $A(t)$, only the long-term trend of $\omega(t)$ is considered in the function of $\omega_{\mathrm{e}}(A)$. However, the $\omega(t)$ of a weakly nonlinear system always contains significant fast-varying components that are large in magnitude. The fast components can be eliminated by removing in advance the fast component contained in $\varphi(t)$ through a least square polynomial fitting on the identified data.

\subsubsection{Determination of instantaneous damping ratio}

The instantaneous damping is dependent on energy dissipation of a vibration system and is often different from case to case. It is generally difficult to determine the instantaneous damping from responses of vibration. In this study, a constant variant method is applied to identify the nonlinear damping ratio of a weakly nonlinear system.
For a linear system, the instantaneous amplitude of a free decay response is written as

$A(t)=\mu_{0} e^{-\xi_{0} \omega_{0} t}$

Equation (42) can be rewritten by

$$
-\frac{1}{\omega_{0}} \ln A(t)+\frac{\ln u_{0}}{\omega_{0}}=\xi_{0} t
$$

Equation (43) is further generalized as

$B(t)+B_{0}=\xi_{0} t$

In a linear system, $B(t)$ is linear with time and the damping ratio is the slope of the linear curve of $B(t)$.

In a nonlinear system, the damping ratio and frequency are amplitude dependent. Similar with the expressions for a linear system in Eq. (42), the instantaneous amplitude of a free decay response is expressed as

$A(t)=\mu_{0} e^{-\xi_{\mathrm{e}}(A) \omega_{\mathrm{e}}(A) t}$

Similarly,

$B(t)+B_{0}=\xi_{\mathrm{e}}(A) t$

where

$$
\begin{aligned}
B(t) & =-\frac{1}{\omega_{\mathrm{e}}(A)} \ln A(t) \\
B_{0}(t) & =-\frac{1}{\omega_{\mathrm{e}}(A)} \ln u_{0}
\end{aligned}
$$

Apparently, in a nonlinear system, $B(t)$ varies nonlinearly with time, and the slope of $B(t)$ is the amplitudedependent damping ratio $\xi_{\mathrm{e}}(A)$ that can be determined by the instantaneous amplitude $A(t)$ identified by the MMWT given in Eq. (46). The amplitude-dependent damping ratio is expressed by

$\xi_{\mathrm{e}}(t)=\frac{\mathrm{d} B(t)}{\mathrm{d} t}=-\frac{1}{\omega_{\mathrm{e}}(t) A(t)} \dot{A}(t)$

Following the above procedure, the nonlinearities of a weakly nonlinear system can be determined and quantified. For the purpose of convenient application, a framework for the identification of the nonlinearities of a weakly nonlinear system is summarized in Fig. 1.

\section{A hybrid aeroelastic-pressure balance (HAPB)}

\subsection{Development of HAPB system}

As mentioned before, a novel HAPB system was devised to simultaneously measure the unsteady wind 


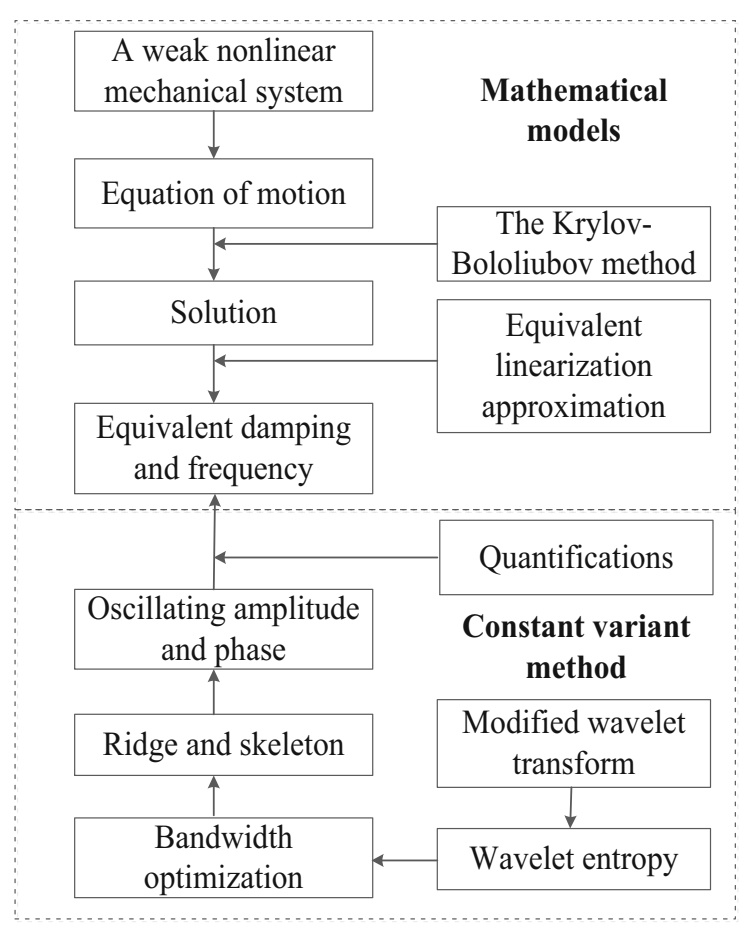

Fig. 1 A framework for the identification of the nonlinearities of a weakly nonlinear system

force and response of a rectangular test model (bridge pylon). The measured wind force including the effect of structural motion is more accurate than that measured from the conventional wind tunnel test techniques (i.e., the HFBB and the SMPSS test techniques). The test model is made of high-performance plastic with a dimension of $50.8 \mathrm{~mm} \times 50.8 \mathrm{~mm} \times 914.4 \mathrm{~mm}$. The test rig is made of high-strength aluminum alloy, and the diameter of the turntable is $60 \mathrm{~cm}$. The elements of the HAPB system are presented in Fig. 2.

In Fig. 2, the test model (E) was fixed with the circular plate (D) by two clamping steels. The circular plate was linked to the circular ring $(\mathrm{C})$ by pivot. The ' $U$ '-shaped connection $(G)$ was connected with (D). The spring, adjusting mass, and the mechanical damper immersed in the oil tank (N) (Figs. 2, 3) were used to adjust the mechanical parameter (i.e., the stiffness and damping) of the system. When the test model (E) is excited by outer force (e.g., wind force), it oscillates with the circular plate (D) and the 'U'-shaped connection. The oscillation of the ' $U$ '-shaped connection was associated with the deformation of the cantilever beam, which was recorded by a strain collection system. The relationship between the observed strain of the can-

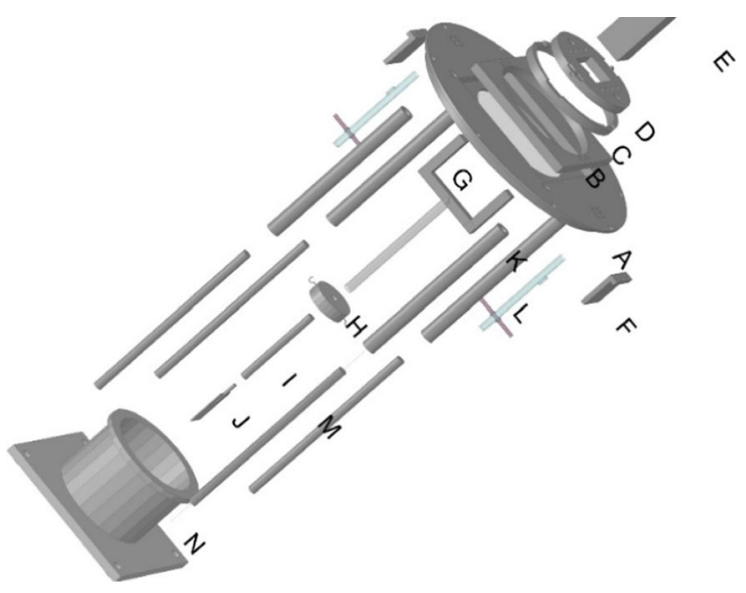

Fig. 2 Assembly schematic diagram of the HAPB system: (A) turntable; (B)—rectangular block; (C)—circular ring; (D) circular plate; (E)—test model; (F)—clamping steel; (G) 'U'-shaped connection; (H)_adjusting mass; (I), (K), (M) connecting rods; (J)—mechanical damper; (L)—cantilever beam; (N)_oil tank

tilever beam and the tip oscillating amplitude of the test model (E) was determined through a method of static calibration. Details of the calibration can be found in a previous study [32]. Accordingly, the time-history response of the test model was obtained. Meanwhile, the wind-induced unsteady pressure of the prism was measured by using pressure taps that connected with the pressure tubes installed in the test model (Fig. 3). There are 72 pressure taps installed on lateral faces of the test model, which were connected with 72 pressure tubes and 12 pressure transducers. The length and diameter of the pressure tube are $1.2 \mathrm{~m}$ and $2 \mathrm{~mm}$, respectively.

The aerodynamic behaviors of inclined square prisms have been investigated comprehensively $[1,33,34]$, due to its practical significance for bridge towers. The prevalence of the inclined structures is due to not only its artistic appearance but also superior load-bearing performance. It should be emphasized that the HAPB system is not only utilized to test vertical prisms, but also inclined prisms by changing different pairs of the clamping steel (F) (Fig. 2). The inclination $\alpha$ of the models ranged from $0^{\circ}$ to $30^{\circ}$ at an interval of $10^{\circ}$ (Fig. 4).

The free decay response tests of the system with vertical and inclined test models were performed in the high wind speed section of the wind tunnel at the CLP Power Wind/Wave Tunnel Facility of the Kong University of Science and Technology. The dimensions of the 

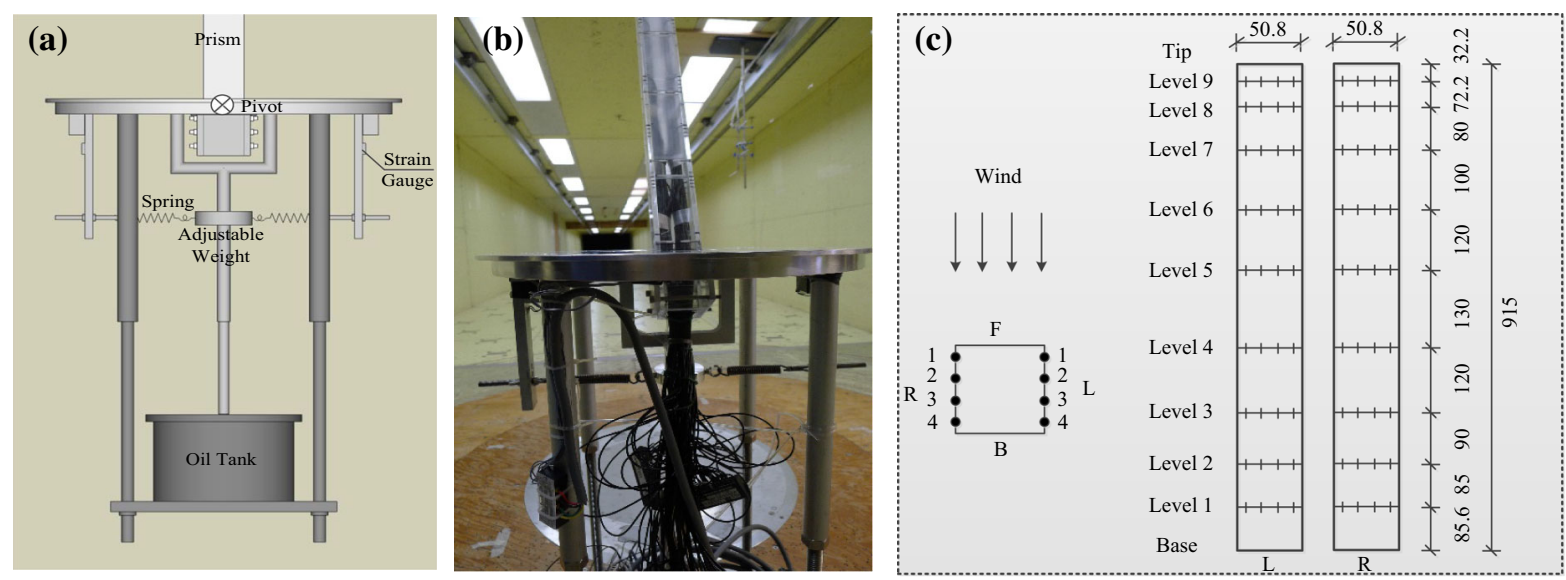

Fig. 3 HAPB system: a schematic diagram; $\mathbf{b}$ test rig and model; $\mathbf{c}$ distribution of pressure taps

Fig. 4 Inclined test models: a schematic diagram; $\mathbf{b}$ an inclined model in a wind tunnel
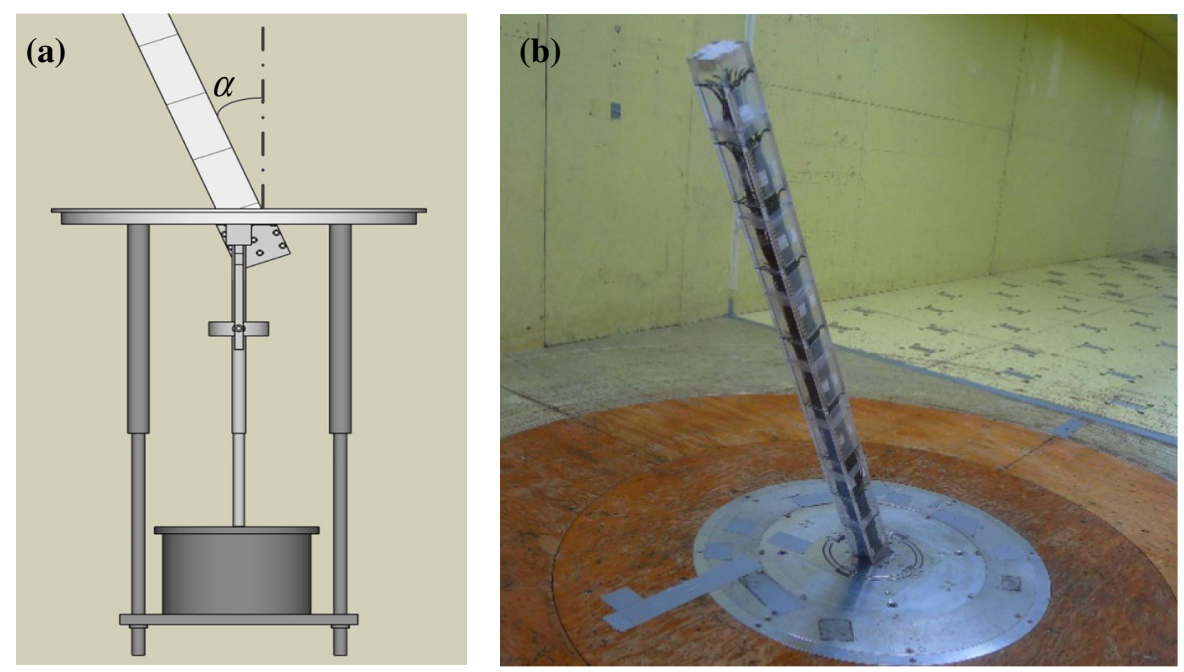

high wind speed test section are $29.2 \mathrm{~m}$ long $\times 3 \mathrm{~m}$ wide $\times 2 \mathrm{~m}$ high. The test model was pivoted to oscillate under excitation. The test model was excited by an initial displacement (around 0.3 times of the model width) in cross-wind direction. The free decay response at the tip end of the test model was recorded, at a sampling frequency of $500 \mathrm{~Hz}$, by using a laser displacement sensor or strain gauges attached in the cantilever beams of the HAPB system (Fig. 3). The model stiffness $k_{s}$ was $441.7 \mathrm{~N} / \mathrm{m}$, and the corresponding equivalent density of the test models was $274 \mathrm{~kg} / \mathrm{m}^{3}$ obtained from static calibrations. The fundamental frequency and the damping ratio of the test models can be determined by the free decay tests of the models. They have been detailed in the following using linear and nonlinear analytical models.

\subsection{System identification: a linear identification}

Using the linear identification model presented in "Appendix A", the undamped frequency and damping ratio of the HAPB system with a vertical test model are determined from a free decay signal (blue line in Fig. 5), and $\omega_{0}=49.26 \mathrm{rad} / \mathrm{s}$ and $\xi_{0}=0.69 \%$, respectively. To verify the effectiveness of the linear identification model, the time-history response was calculated from Eq. (A2) and compared with that directly measured from the HAPB system with a vertical test model. Figure 5 displays notable discrepancies between the free decay response computed by the linear model and that directly measured from the HAPB system. The discrepancies are ascribed to the poor estimation of the long-duration of phase and amplitude. Figure 5 also 
Fig. 5 Comparison of free decay response predicted by the linear model with that measured from the HAPB system with a vertical test model

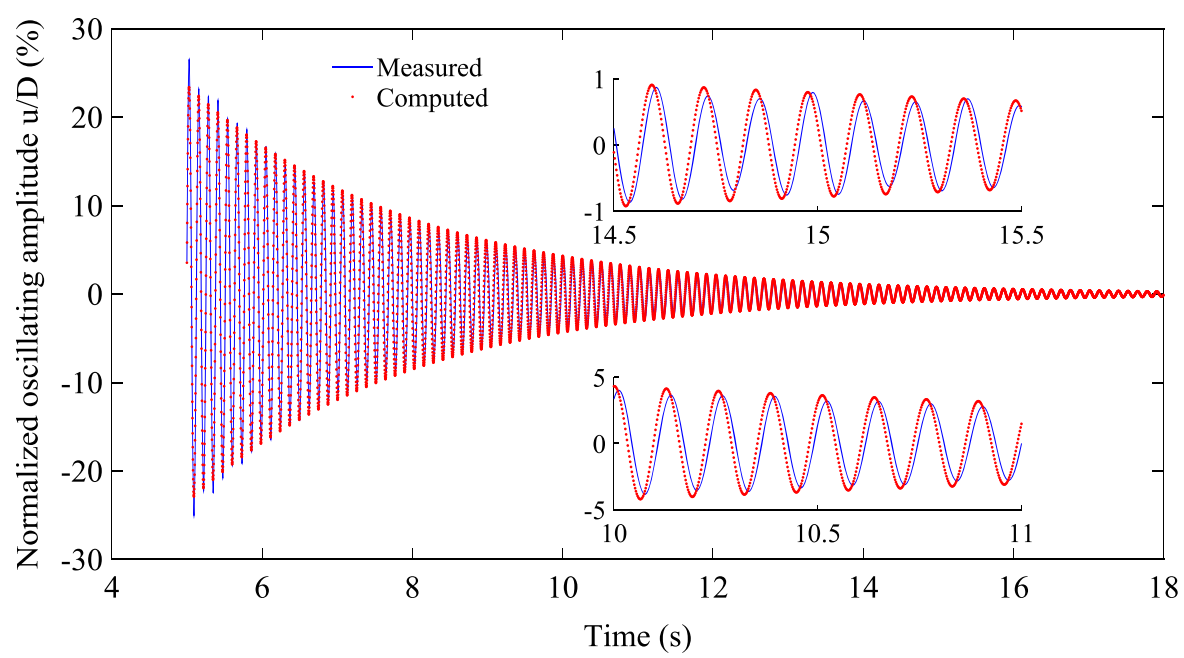

shows that the phase computed by the linear model does not agree well with that directly measured from the HAPB system, and the computed successive peaks are notably larger than the directly measured. This was induced by the considerable effect of the mechanical nonlinearities of the HAPB. These physical nonlinearities may be caused by friction between joints and interfaces and nonlinearities of the helical springs (Figs. 2, 3 ). Also, the pressure tubes installed in the test model would oscillate with the oscillation of the test model, and this may generate nonlinearities of the HAPB system to some extent. The above illustration suggests that the physical nonlinearity would cause a time-varying or amplitude-dependent natural frequency, and a conventional linear model is limited to predict the timevarying or amplitude-dependent mechanical nonlinear characteristics. It is necessary to develop a nonlinear analytical model to precisely identify the mechanical nonlinearities of the HAPB system, which should be precise enough to predict the free decay response of the HAPB system.

\section{Verification of the identification for the physical nonlinearities of the HAPB system}

The nonlinearities of the HAPB system with a vertical test model ( $\alpha=0^{\circ}$, in Fig. 3) will be identified by the proposed method (Sect. 2), and will be validated by a time domain method and the Newmark- $\beta$ method. Then, the nonlinearities of the HAPB system with inclined test models will be identified in a similar way.

\subsection{Analytical process using the modified Morlet} wavelet transform

The free decay response of the HAPB system with a vertical test model is observed in Sect. 2.2 (blue line in Fig. 5). The amplitude of the response using the MMWT given by Eq. (34) for bandwidth parameter $f_{\mathrm{b}}=2$ is presented in Fig. 6. It shows that the timefrequency resolution is not good. To ensure the timefrequency resolution, the optimal bandwidth $f_{\mathrm{b}}$ of the MMWT is determined by minimization the wavelet entropy defined in Eq. (36). It is observed in Fig. 7 that the optimal $f_{\mathrm{b}}$ is found as $f_{\mathrm{b}}=49$ leading to a minimum wavelet entropy. The amplitude of the MMWT

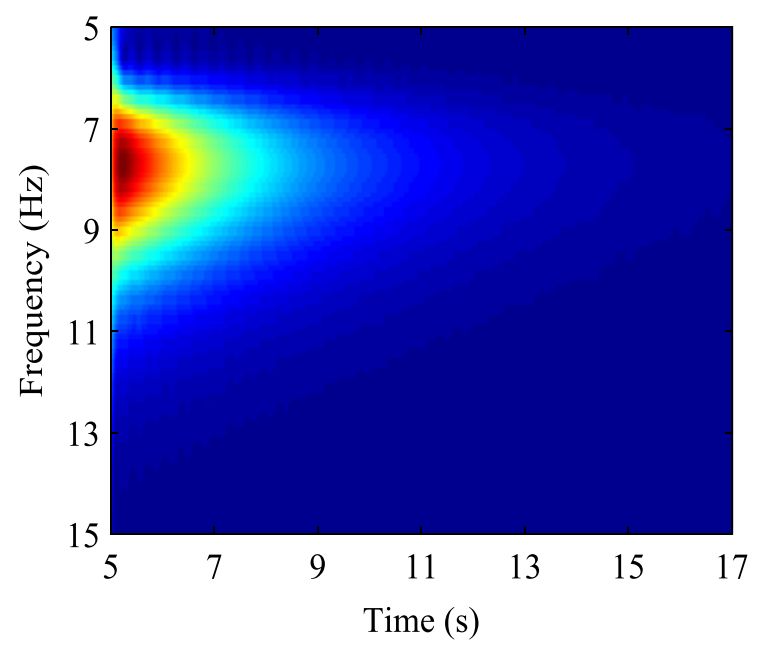

Fig. 6 Amplitude of the MMWT for $f_{\mathrm{b}}=2$ 


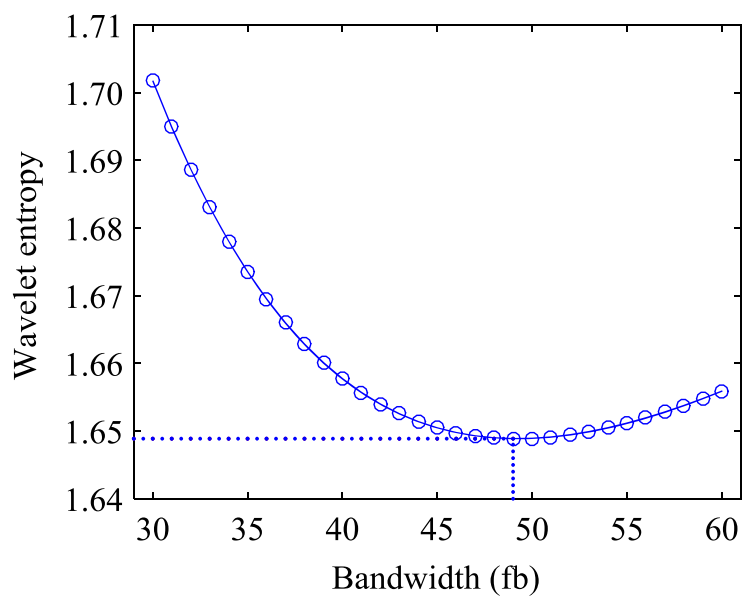

Fig. 7 Entropy of the MMWT

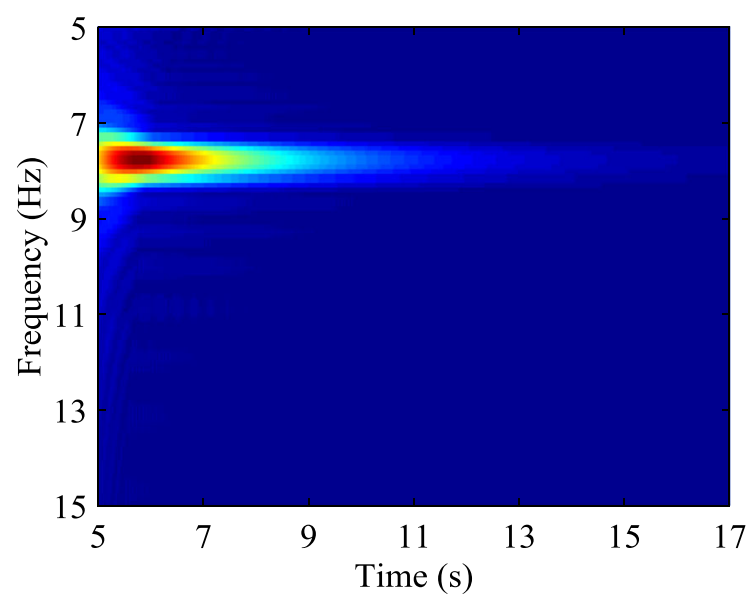

Fig. 8 Amplitude of the MMWT for $f_{\mathrm{b}}=49$

for $f_{\mathrm{b}}=49$ is presented in Fig. 8. It is found that the time-frequency resolution is well improved.

The coefficient of the MMWT is then determined from Fig. 8, and the ridge and skeleton of the MMWT are extracted from Eq. (41). As a consequence, the envelope $A(t)$ of the signal is determined. The comparisons of the envelope predicted by the MMWT and that directly observed are given in Fig. 9. It shows that the envelope predicted by the MMWT for $f_{\mathrm{b}}=49$ is in better agreement with the directly observed than that for $f_{\mathrm{b}}=2$. Moreover, the plots of the envelope predicted by the MMWT for $f_{\mathrm{b}}=49$ and the directly observed coincide well with each other apart from the beginning of the data due to significant 'end effect'. This effect has been studied in a number of previous studies [35-37]. Note that the effect can be reduced by

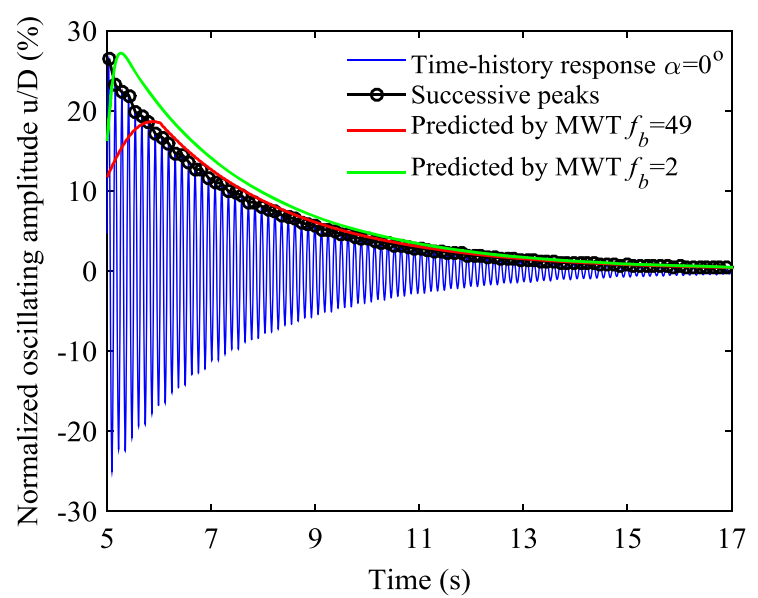

Fig. 9 Comparisons of the envelope predicted by the MMWT and that directly observed

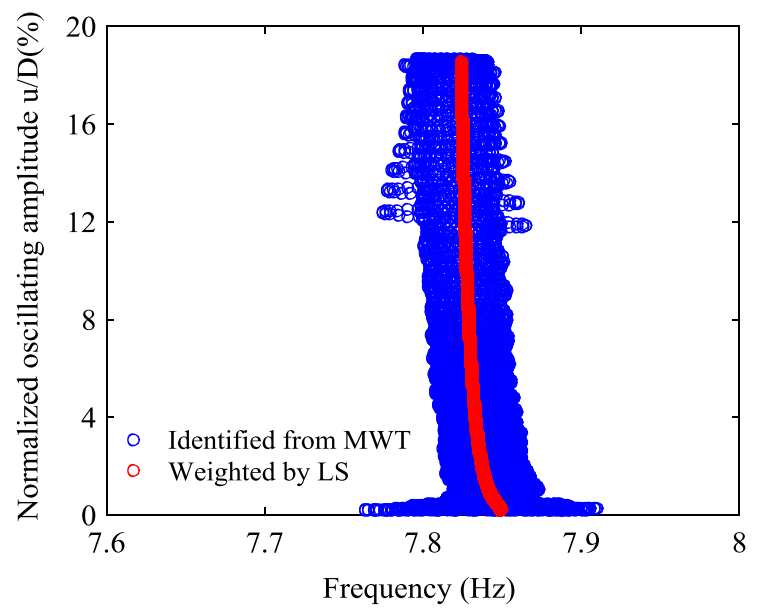

Fig. 10 Instantaneous frequency and response of the signal

adding zeros or by adding negative values of the signal at the end region [35], or it could be fitted out as long as the signal is long enough. We adopt the latter method to reduce the effect, and the identification of nonlinearities using this method will be verified.

The phase of the signal is determined by Eq. (36). It is found in Fig. 10 that the instantaneous frequency identified from the MMWT has significant fast-varying components. As mentioned before, the fast-varying components contained in $\varphi(t)$ should be removed in advance through a least square (LS) polynomial fitting. The instantaneous frequency and response of the signal identified from the MMWT and that removing the fast-varying component by the LS fitting are plotted in Fig. 10. 


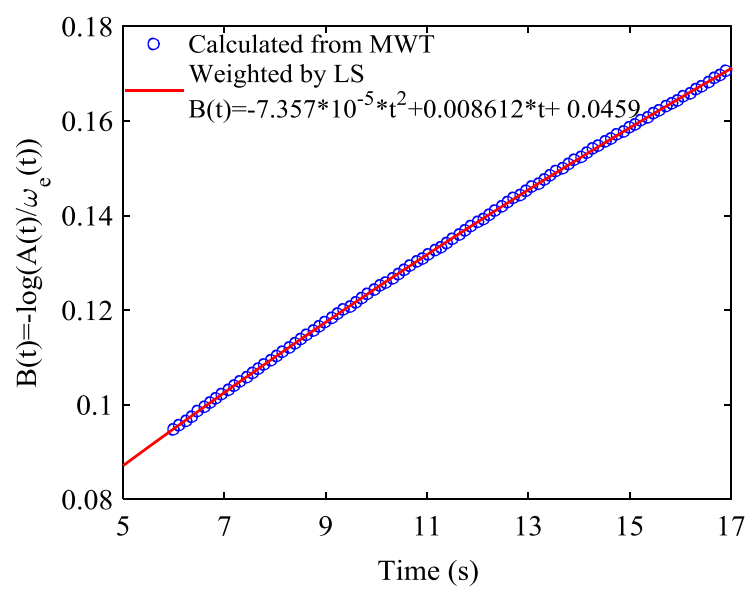

Fig. $11 B(t)$ calculated from MMWT and weighted by the LS

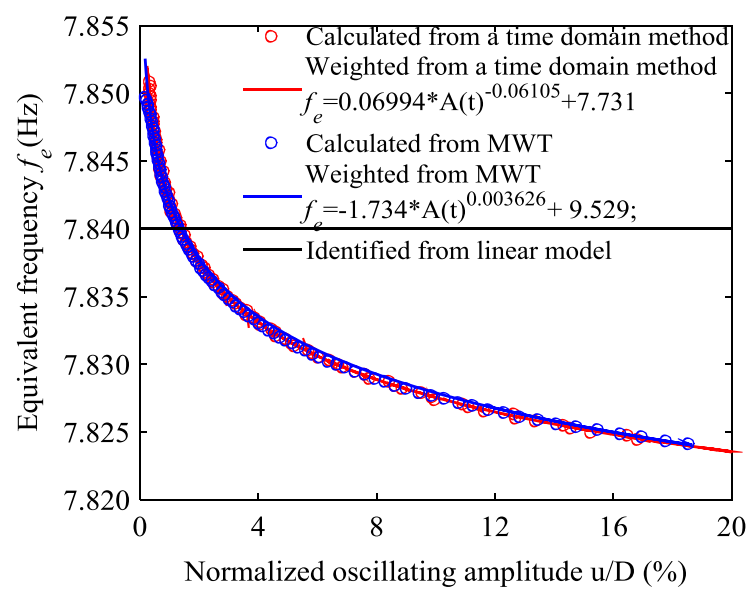

Fig. 12 Identifications and comparisons of the nonlinear frequency of the HAPB system

Figure 11 plots $B(t)$ given by Eq. (47). It is observed that the slope of $B(t)$ varies slowly with time, indicating that the damping ratio identified by Eq. (49) would also varies slowly with time. Using the LS method, $B(t)$ is weighted by a polynomial, which is readily utilized to obtain the damping ratio of the signal.

\subsection{Nonlinear physical frequency and damping ratio}

The nonlinear frequency $f_{\mathrm{e}}=\omega_{\mathrm{e}} / 2 \pi$ and damping ratio of the HAPB system with a vertical test model are identified by the MMWT method following the framework in Fig. 1. Figure 12 presents the nonlinear frequency of the system. It is observed that the nonlinear frequency of the system decreases slowly with increasing oscillating response, suggesting that

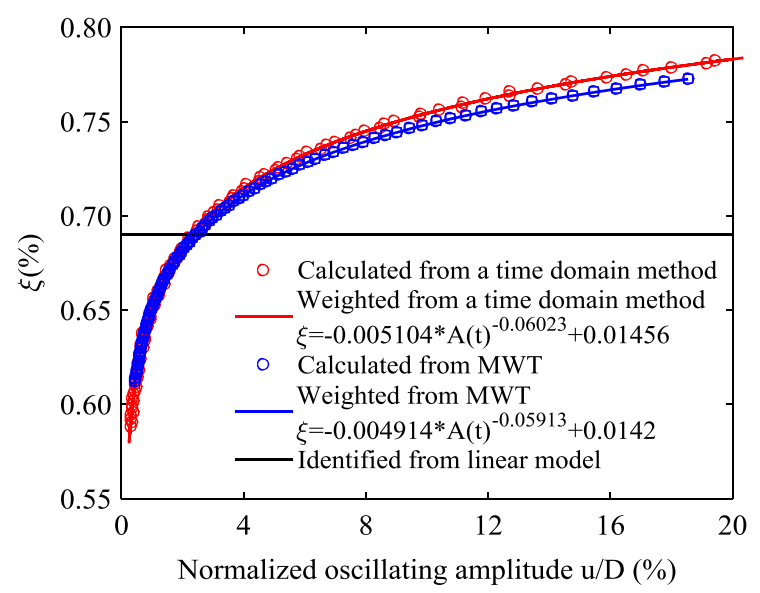

Fig. 13 Identifications and comparisons of the nonlinear damping of the HAPB system

the stiffness of the HAPB system is a weakly nonlinear system. Figure 12 also plots the constant frequency identified by the linear model presented in "Appendix A". Comparing the nonlinear frequency with the constant frequency indicates that the slow varying behavior of the frequency is filtered out by the linear model, and the complex behavior of phase modulation is therefore not precisely predicted (in Fig. 5). Moreover, the nonlinear frequency identified by the MMWT is compared with that identified by a time domain method detailed in "Appendix B" (Figs. 16, 17). It is noteworthy that the nonlinear frequency identified by the MMWT is in good agreement with that identified by the time domain method, suggesting that the proposed identification method for the identification of the nonlinear frequency of the HAPB system is reliable and the identified nonlinear frequency of the system, $f_{\mathrm{e}}=-1.734 A(t)^{0.003626}+9.529$, could be utilized for further analysis.

Figure 13 plots the nonlinear damping of the HAPB system with a vertical test model. It is noted that the nonlinear damping increases gradually with amplitude of oscillation. The damping identified from the linear model presented in "Appendix A" is linear, and can only describe approximately the dissipative behaviors of the nonlinear oscillating HAPB system. Furthermore, the nonlinear damping identified by a time domain method is also plotted and compared with the nonlinear damping identified by the MMWT method. Figure 13 shows that the nonlinear damping identified by the MMWT agrees well with that obtained from the time domain method defined in "Appendix B" (Figs. 16, 
17). There exist slight discrepancies at large oscillations (i.e., $u / D=12--20 \%$ ), which may be attribute to the errors caused by curve fitting, such as the errors in the curve fitting of $B(t), f_{\mathrm{e}}$, etc. But the discrepancy is so small (the maximum is around $1.27 \%$ ) that it is negligible. This suggests that the proposed identification method is reliable and the identified nonlinear damping of the HAPB system, $\xi=-0.004914 A(t)^{-0.05913}+$ 0.0142 , could be utilized for further analysis.

The nonlinearities of the HAPB system, depicted in Figs. 12 and 13, may arise from a series of uncertainties of the system. Generally, the nonlinear frequency of the system is due to the nonlinear elastic effect of
4.3 Verification of the identified nonlinear physical frequency and damping ratio

To further validate the nonlinear frequency (Fig. 12) and nonlinear damping (Fig. 13) of the HAPB system with a vertical test model, identified by the MMWT method, the free decay response of the model is numerically calculated by the Newmark- $\beta$ method and compared with the directly observed in Fig. 5.

By solving the equation of motion of Eq. (33) using theNewmark- $\beta$ method, the numerically calculated response of the displacement, velocity and acceleration of the HAPB system at time step $t_{i+1}$ is given as

$$
\left\{\begin{array}{l}
u_{t_{i+1}}=\left[\left(a_{0} u_{t_{i}}+a_{2} \dot{u}_{t_{i}}+a_{3} \ddot{u}_{t_{i}}\right)+2 \xi_{\mathrm{e}}\left(t_{i}\right) \omega_{\mathrm{e}}\left(t_{i}\right)\left(a_{1} u_{t_{i}}+a_{4} \dot{u}_{t_{i}}+a_{3} \ddot{u}_{t_{i}}\right)\right] /\left[\omega_{\mathrm{e}}^{2}\left(t_{i}\right)+a_{0}+2 a_{1} \xi_{\mathrm{e}}\left(t_{i}\right) \omega_{\mathrm{e}}\left(t_{i}\right)\right] \\
\dot{u}_{t_{i+1}}=\dot{u}_{t_{i}}+a_{6} \ddot{u}_{t_{i}}+a_{7} \ddot{u}_{t_{i+1}} \\
\ddot{u}_{t_{i+1}}=a_{0}\left(u_{t_{i+1}}-u_{t_{i}}\right)-a_{2} \dot{u}_{t_{i}}-a_{3} \ddot{u}_{t_{i}}
\end{array}\right.
$$

restoring stiffness, and the nonlinear damping of the system is attributed to a complicated energy dissipative mechanism. More specifically, the physical sources of the nonlinearities of the HAPB system are briefly summarized as: (1) the viscous damping generated by the interaction between the sounding still air and the test model; (2) the friction between the components of the HAPB system at joints, connections, and interfaces (in Fig. 2); (3) the viscous damping generated by the oscillation of the pressure tube attached on the inside surface of the test model (in Figs. 3, 4); (4) the material damping of the HAPB system, due to complex molecular interactions within the material; (5) the nonlinear damping introduced by additional dampers, such as air or oil damper in Fig. 3. where

$$
\begin{aligned}
a_{0} & =\frac{1}{\beta \cdot \Delta t^{2}}, a_{1}=\frac{\gamma}{\beta \cdot \Delta t}, a_{2}=\frac{1}{\beta \cdot \Delta t}, \\
a_{3} & =\frac{1}{2 \beta}-1, a_{4}=\frac{\gamma}{\beta}-1, a_{5}=\left(\frac{\gamma}{2 \beta}-1\right) \cdot \Delta t, \\
a_{6} & =(1-\gamma) \cdot \Delta t, a_{7}=\gamma \cdot \Delta t \\
\gamma & =0.5, \beta=1 / 6
\end{aligned}
$$

The initial condition $u_{0}$ is defined as the first value of the observed time-history response of the HAPB system (in Fig. 4), and $\dot{u}_{0}=0$. The time step $\Delta t=0.002$ which is the inverse of the sampling frequency $(500 \mathrm{~Hz})$.

The comparison of the time-history free decay response computed from Eq. (50) and that directly observed is presented in Fig. 14. It demonstrates that
Fig. 14 Comparison of the time-history free decay response computed from Eq. (50) and that directly observed

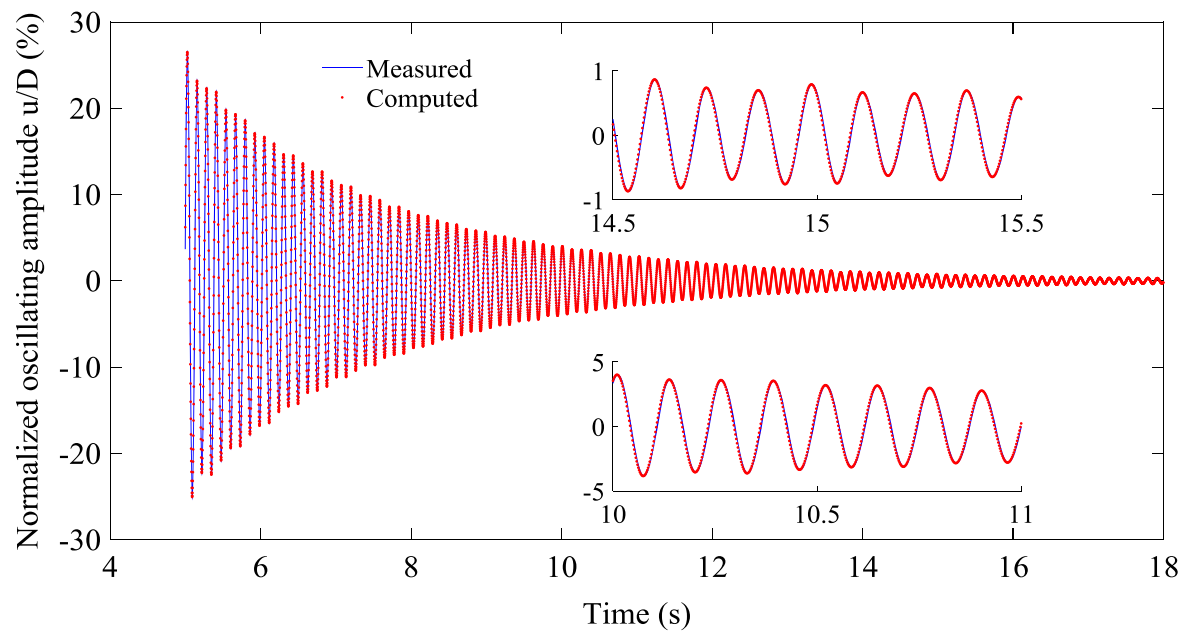



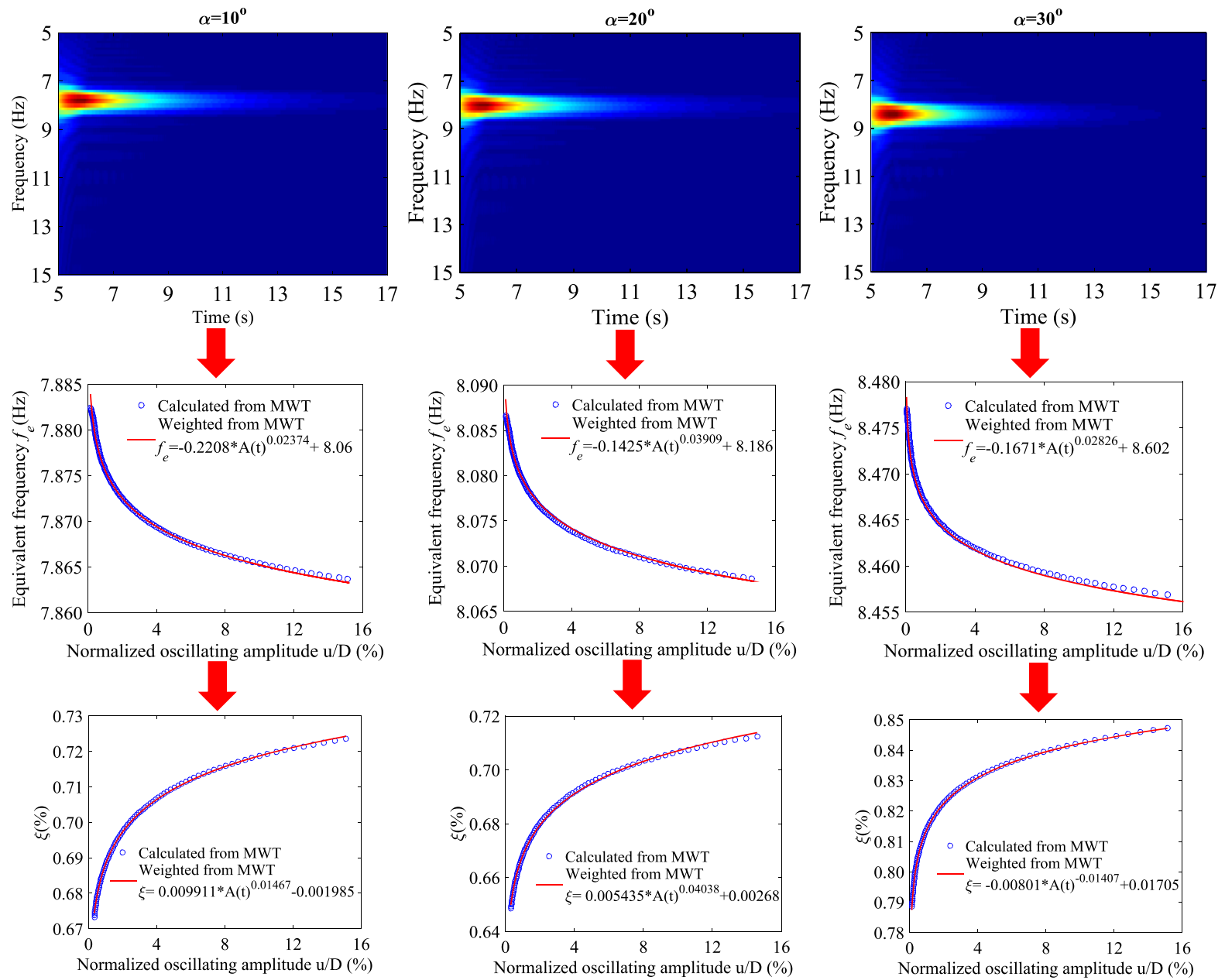

Fig. 15 Physical nonlinearities of the HAPB system with inclined test models, identified from the MMWT

the computed response based on the identified nonlinear frequency and damping is coincide with the directly measured, suggesting that the nonlinearities of the HAPB system identified by the MMWT are reliable and accurate. Comparing the result in Fig. 14 with that in Fig. 4 indicates that the relatively poor agreement using the linear model is attribute to the ignorance of the nonlinearities of the HAPB system.

\section{Nonlinearities of the HAPB system: with inclined test models}

As mentioned before, the HAPB system can also be used to test models with different inclinations (Fig. 4). The nonlinearities of the HAPB system with a vertical system have been identified by the MMWT and verified in the previous section. The nonlinearities of the system with inclined test models are determined in a similar way and are presented in Fig. 15. It shows that the nonlinear frequency and damping ratio of the system with inclined test models vary with the inclination of the models. This may be attribute to the different physical sources induced by different inclinations: (1) the different interactions between the sounding still air and the test model; (2) the different frictions between the components of the HAPB system at joints, connections, and interfaces. But the trend is identical to that of the HAPB system with a vertical test model. Also, both the nonlinear frequency and damping ratio of the system vary slowly with increasing oscillating response, indicating the stiffness and damping of the system with inclined test models are weakly nonlinear systems. According to 
Fig. 15, the expressions of the nonlinear frequency and damping ratio of the system weighted by the MMWT are summarized in Eqs. (53) and (54), respectively.

$$
\begin{aligned}
& f_{\mathrm{e}}=\left\{\begin{array}{l}
-0.2208 A(t)^{0.02374}+8.06, \quad \alpha=10^{0} \\
-0.1425 A(t)^{0.03909}+8.186, \quad \alpha=20^{0} \\
-0.1671 A(t)^{0.02826}+8.602, \quad \alpha=30^{0}
\end{array}\right. \\
& \xi_{\mathrm{e}}=\left\{\begin{array}{l}
0.009911 A(t)^{0.01467}-0.001985, \quad \alpha=10^{0} \\
0.005435 A(t)^{0.04038}+0.00268, \quad \alpha=20^{0} \\
-0.00801 A(t)^{-0.01407}+0.01705, \quad \alpha=30^{0}
\end{array}\right.
\end{aligned}
$$

\section{Concluding remarks}

This study has proposed an improved method for determining physical nonlinearities of weakly nonlinear spring-suspension system and successfully applied to a novel hybrid aeroelastic-pressure balance (HAPB) system used in wind tunnel, which can be used for simultaneously obtaining the unsteady wind pressure and aeroelastic response of a test model. The identification was verified through comparing the identifications with those identified by a time domain method. The main findings are concluded: (1) The frequency and damping of the HAPB system identified by a linear model are constant and will lead to large discrepancies in response predictions due to the ignorance of the slow varying characteristics of the system. The nonlinearities of the system have to be considered; (2) the proposed identification method and the analytical scheme are precise and reliable in identifying the nonlinearities of the system. They are able to predict the long-duration free decay response of the system; (3) the identified nonlinearities of the system with inclined test models vary with the inclination of the models, which may be attribute to the different physical sources induced by the inclination.

\footnotetext{
Acknowledgements The work described in this paper was supported by 111 project of China (Grant No.: B18062), Natural Science Foundation of Chongqing, China (Grant No.: cstc2019jcyjmsxm0639), Fundamental Research Funds for the Central Universities (Project No.: 2019CDXYTM0032), Research Grants Council of the Hong Kong Special Administrative Region, China (Project No. 16242016). Also, the authors appreciate the use of the testing facility, as well as the technical assistance provided by the CLP Power Wind/Wave Tunnel Facility at the Hong Kong University of Science and Technology. The authors would also like to express our sincere thanks to the Design and Manufacturing Services Facility (Electrical and Mechanical Fabrication Unit) of the Hong Kong University of Science and Technology for their help in manufacturing the test rig of the pressure-aeroelastic hybrid wind tunnel test system.
}

Open Access This article is distributed under the terms of the Creative Commons Attribution 4.0 International License (http:// creativecommons.org/licenses/by/4.0/), which permits unrestricted use, distribution, and reproduction in any medium, provided you give appropriate credit to the original author(s) and the source, provide a link to the Creative Commons license, and indicate if changes were made.

\section{Appendix A: Linear identification model}

It is common in practice to utilize a linear mechanical model to identify the physical nonlinear stiffness and damping of a wind tunnel test system from a time series of free decay response. The linear model is also applied to identify the parameters of the HAPB system. In the linear model, the parameters are assumed to be constant and invariant with time, and the equation of motion of the system is therefore expressed as a time invariant, second-order linear system

$\ddot{u}(t)+2 \xi_{0} \omega_{0} \dot{u}(t)+\omega_{0}^{2} u(t)=f(t)$

where $u(t), \dot{u}(t)$ and $\ddot{u}(t)$ are the oscillating amplitude, velocity and acceleration of the test model, respectively; $\xi_{0}$ is the equivalent viscous damping ratio; $\omega_{0}$ is the undamped natural circular frequency; $f(t)$ is the outer excitation (i.e., wind force).

The physical parameters in Eq. (A1) are identified via a logarithmic decrement method from a free decay response with $f(t)=0$, and the free decay response is expressed as

$$
\begin{aligned}
& u(t)=u_{0} e^{-\xi_{0} \omega_{0} t} \sin \left(\omega_{\mathrm{d}} t+\varphi\right) \\
& \omega_{\mathrm{d}}=\omega_{0} \sqrt{1-\xi_{0}}
\end{aligned}
$$

where $\omega_{\mathrm{d}}$ is damped natural frequency.

The constant viscous damping ratio is determined by the logarithm of the ratio of successive peaks and is written as

$$
\xi_{0}=\frac{1}{\sqrt{1+\left(2 \pi N / \sum_{i=1}^{N} \frac{u_{i}}{u_{i+1}}\right)^{2}}}
$$

where $N$ is the total number of the successive positive peaks (e.g., $u_{i}, u_{i+1}$ ).

The damped natural frequency $\omega_{\mathrm{d}}$ can be derived from the time intervals of successive peaks and is expressed as

$$
\omega_{\mathrm{d}}=\frac{1}{N} \sum_{i=1}^{N} \frac{2 \pi}{t_{i+1}-t_{i}}
$$


where $t_{i}$ corresponds to time of occurrence of the successive peaks. The undamped frequency is then obtained from Eq. (A3).

$\omega_{0}=\frac{\omega_{\mathrm{d}}}{\sqrt{1-\xi_{0}^{2}}}$

\section{Appendix B: A time domain method for comparison}

A time domain method was proposed and verified in a previous study [10]. This method is utilized to determine the instantaneous envelope and phase of a signal. The process is briefly introduced infra.

Equation (26) is rewritten as

$$
\dot{u}(t)=-A(t) \omega_{0} \sin \left[\omega_{0} t+\varphi(t)\right]+o(\varepsilon)
$$

From Eq. (B1), the responses of displacement and velocity are expressed as

$$
A(t) e^{i\left[\omega_{0} t+\varphi(t)\right]}=u(t)-i \frac{\dot{u}(t)}{\omega_{0}}
$$

Projecting Eq. (B2) in Cartesian coordinate into the coordinate of a complex plane, the instantaneous envelope and phase of a signal can be obtained as

$$
\begin{aligned}
& A(t)=\sqrt{u(t)^{2}+\left[\frac{\dot{u}(t)}{\omega_{0}}\right]^{2}} \\
& \varphi(t)=-\arctan \frac{\dot{u}(t)}{\omega_{0} u(t)}
\end{aligned}
$$

The instantaneous circular frequency is therefore determined by the first-order differentiation of Eq. (B4). We have

$\varphi(t)=\frac{\mathrm{d} \varphi(t)}{\mathrm{d} t}=\frac{\dot{u}(t)^{2}-\ddot{u}(t) u(t)}{\omega_{0} A(t)^{2}}$

where $\dot{u}(t)$ and $\ddot{u}(t)$ are the responses of the velocity and the acceleration of the signal, and they can be determined by a fourth-order central difference method and expressed as

$$
\begin{aligned}
& \dot{u}_{i}=\left\{\begin{array}{l}
\frac{-3 u_{i}+4 u_{i+1}-u_{i+2}}{2 \Delta t}, 1 \leq i \leq 2 \\
\frac{u_{i-2}-8 u_{i-1}+8 u_{i+1}-u_{i+2}}{12 \Delta t}, 2<i \leq n-2 \\
\frac{3 u_{i}-4 u_{i+1}+u_{i+2}}{2 \Delta t}, n-2<i \leq n
\end{array}\right. \\
& \ddot{u}_{i}=\left\{\begin{array}{l}
\frac{u_{i}-2 u_{i+1}+u_{i+2}}{\Delta t^{2}}, 1 \leq i \leq 2 \\
\frac{-u_{i-2}-16 u_{i-1}-30 u_{i}+16 u_{i+1}-u_{i+2}}{12 \Delta t^{2}}, 2<i \leq n-2 \\
\frac{u_{i}-2 u_{i-1}+u_{i-2}}{\Delta t^{2}}, n-2<i \leq n
\end{array}\right.
\end{aligned}
$$

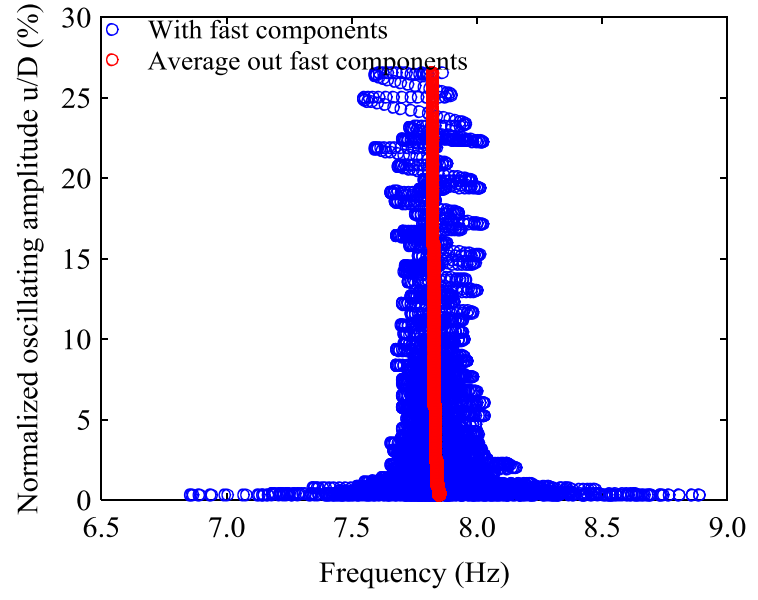

Fig. 16 The instantaneous frequency of the free decay response using the time domain method

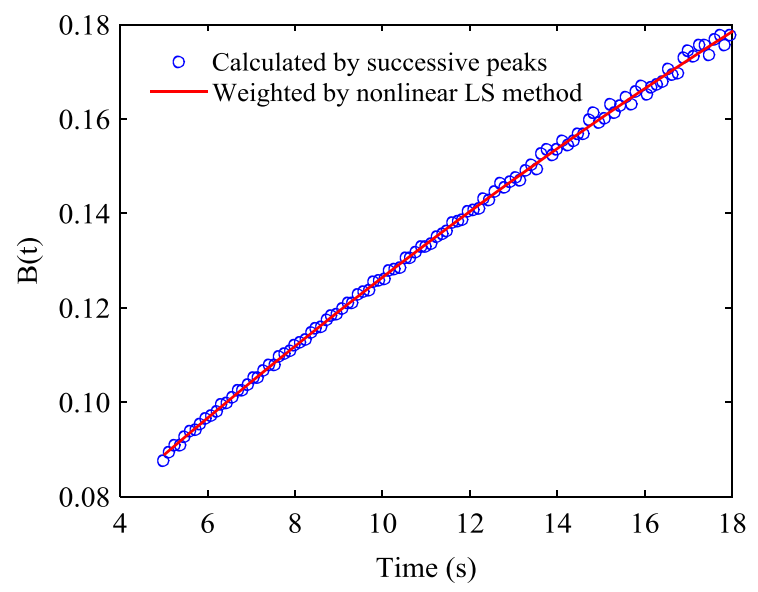

Fig. $17 B(t)$ calculated from the time domain method and weighted by LS

It should be mentioned that, before using the time domain method, the low-pass signal needs to be removed in advance by a low-pass filter. Then, the amplitude-dependent circular frequency is obtained as

$\omega_{\mathrm{e}}(t)=\frac{\mathrm{d} \varphi_{\mathrm{e}}(t)}{\mathrm{d} t}$

where $\varphi_{\mathrm{e}}(t)$ is the long-term trend of identified $\varphi(t)$.

The instantaneous damping ratio of a signal is determined by the constant variant method introduced in Sect. 2.2.3, and the expressions are the same with those in Sect. 2.2.3.

Using the time domain method and Eq. (B8), the instantaneous frequency of the free decay response of 
the HAPB system with a vertical test model is determined as shown in Fig. 16.

The $B(t)$ of the signal calculated from the time domain method and weighted by LS is presented in Fig. 17.

From Figs. 16 and 17, the nonlinear frequency and damping ratio of the HAPB system are therefore determined by Eqs. (B8) and (49) based on the identified instantaneous envelope and phase of the signal using the time domain method. They are presented in Figs. 12 and 13 , respectively, for the purpose of comparison.

\section{References}

1. Chen, Z.S., Tse, K.T., Hu, G., Kwok, K.C.S.: Experimental and theoretical investigation of galloping of transversely inclined slender prisms. Nonlinear Dyn. 91(2), 1023-1040 (2018)

2. Diana, G., Resta, F., Belloli, M., Rocchi, D.: On the vortex shedding forcing on suspension bridge deck. J. Wind Eng. Ind. Aerodyn. 94(5), 341-363 (2006)

3. Faltinsen, O.M.: Hydrodynamics of High-Speed Marine Vehicles. Cambridge University Press, Cambridge (2005)

4. Sarkar, P.P., Caracoglia, L., Haan Jr., F.L., Sato, H., Murakoshi, J.: Comparative and sensitivity study of flutter derivatives of selected bridge deck sections, part 1: analysis of inter-laboratory experimental data. EngCambridge Struct. 31(1), 158-169 (2009)

5. Staszewski, W.: Identification of non-linear systems using multi-scale ridges and skeletons of the wavelet transform. J. Sound Vib. 214(4), 639-658 (1998)

6. Feldman, M.: Investigation of the natural vibrations of machine elements using the Hilbert transform. Sov. Mach. Sci. 2(0739-8999), 3 (1985)

7. Yim, S.: Parameter identification of nonlinear ocean mooring systems using the Hilbert transform. J. Offshore Mech. Arct. Eng. 118, 29 (1996)

8. Feldman, M.: Non-linear free vibration identification via the Hilbert transform. J. Sound Vib. 208(3), 475-489 (1997)

9. Feldman, M.: Non-linear system vibration analysis using Hilbert transform-I. Free vibration analysis method'Freevib'. Mech. Syst. Signal Process. 8(2), 119-127 (1994)

10. Gao, G., Zhu, L.: Nonlinearity of mechanical damping and stiffness of a spring-suspended sectional model system for wind tunnel tests. J. Sound Vib. 355, 369-391 (2015)

11. Feldman, M.: Hilbert transform in vibration analysis. Mech. Syst. Signal Process. 25(3), 735-802 (2011)

12. Huang, N.E., Shen Z., Long S.R., Wu M.C., Shih H.H., Zheng Q., Yen N.-C., Tung C.C., Liu, H.H.: The empirical mode decomposition and the Hilbert spectrum for nonlinear and non-stationary time series analysis. In: Proceedings of the royal society of London A: mathematical, physical and engineering sciences, The Royal Society (1998)

13. Feldman, M., Braun, S.: Identification of non-linear system parameters via the instantaneous frequency: application of the Hilbert transform and Wigner-Ville techniques. In: Proceedings-SPIE the international society for optical engineering, SPIE International Society For Optical (1995)

14. Spina, D., Valente, C., Tomlinson, G.: A new procedure for detecting nonlinearity from transient data using the Gabor transform. Nonlinear Dyn. 11(3), 235-254 (1996)

15. Ta, M.-N., Lardiès, J.: Identification of weak nonlinearities on damping and stiffness by the continuous wavelet transform. J. Sound Vib. 293(1), 16-37 (2006)

16. Basu, B., Nagarajaiah, S., Chakraborty, A.: Online identification of linear time-varying stiffness of structural systems by wavelet analysis. Struct. Health Monit. 7(1), 21-36 (2008)

17. Delprat, N., Escudié, B., Guillemain, P., Kronland-Martinet, R., Tchamitchian, P., Torresani, B.: Asymptotic wavelet and Gabor analysis: extraction of instantaneous frequencies. IEEE Trans. Inf. Theory 38(2), 644-664 (1992)

18. Peng, Z., Peter, W.T., Chu, F.: An improved Hilbert-Huang transform and its application in vibration signal analysis. J. Sound Vib. 286(1), 187-205 (2005)

19. Thothadri, M., Casas, R., Moon, F., D'andrea, R., Johnson, C.: Nonlinear system identification of multi-degree-offreedom systems. Nonlinear Dyn. 32(3), 307-322 (2003)

20. Soize, C., Le Fur, O.: Modal identification of weakly nonlinear multidimensional dynamical systems using a stochastic linearization method with random coefficients. J. Mech. Syst. Signal Process. 11(1), 37-49 (1997)

21. Crawley, E.F., Aubert, A.C.: Identification of nonlinear structural elements by force-state mapping. AIAA J. 24(1), 155-162 (1986)

22. Allen, M.S., Sumali, H., Epp, D.S.: Piecewise-linear restoring force surfaces for semi-nonparametric identification of nonlinear systems. Nonlinear Dyn. 54(1), 123-135 (2008)

23. Chen, Z., Li, H., Wang, X., Yu, X., Xie, Z.: Internal and external pressure and its non-Gaussian characteristics of long-span thin-walled domes. Thin Walled Struct. 134, 428-441 (2019)

24. Chen, Z.-S., Tse, K., Kwok, K., Kareem, A.: Aerodynamic damping of inclined slender prisms. J. Wind Eng. Ind. Aerodyn. 177, 79-91 (2018)

25. Harris, C.M., Piersol, A.G.: Harris' Shock and Vibration Handbook, vol. 5. McGraw-Hill, New York (2002)

26. Yu, P.: Analysis on double Hopf bifurcation using computer algebra with the aid of multiple scales. Nonlinear Dyn. 27(1), 19-53 (2002)

27. Dittman, E., Adams, D.E.: Identification of cubic nonlinearity in disbonded aluminum honeycomb panels using single degree-of-freedom models. Nonlinear Dyn. 81(1-2), 1-11 (2015)

28. Benedettini, F., Zulli, D., Vasta, M.: Nonlinear response of SDOF systems under combined deterministic and random excitations. Nonlinear Dyn. 46(4), 375-385 (2006)

29. González-Cruz, C., Jáuregui-Correa, J., DomínguezGonzález, A., Lozano-Guzmán, A.: Effect of the coupling strength on the nonlinear synchronization of a single-stage gear transmission. Nonlinear Dyn. 85(1), 123-140 (2016)

30. Lin, J., Qu, L.: Feature extraction based on Morlet wavelet and its application for mechanical fault diagnosis. J. Sound Vib. 234(1), 135-148 (2000) 
31. Lopes, A.M., Machado, J.T.: Integer and fractional-order entropy analysis of earthquake data series. Nonlinear Dyn. 84(1), 79-90 (2016)

32. Hu, G.: Galloping of an inclined square cylinder. Doctoral Dissertation: The Hong Kong University of Science and Technology: Hong Kong (2015)

33. Hu, G., Tse, K., Kwok, K., Chen, Z.: Pressure measurements on inclined square prisms. Wind Struct. 21(4), 383-405 (2015)

34. Chen, Z.S., Tse, K.T., Kwok, K.C.S., Kareem, A.: Aerodynamic damping of inclined slender prisms. J. Wind Eng. Ind. Aerodyn. 177, 79-91 (2018)

35. Kijewski, T., Kareem, A.: Wavelet transforms for system identification in civil engineering. Comput. Aided Civ. Infrastruct. Eng. 18(5), 339-355 (2003)
36. Boltežar, M., Slavič, J.: Enhancements to the continuous wavelet transform for damping identifications on short signals. Mech. Syst. Signal Process. 18(5), 1065-1076 (2004)

37. Zheng, D., Chao, B., Zhou, Y., Yu, N.: Improvement of edge effect of the wavelet time-frequency spectrum: application to the length-of-day series. J. Geodesy 74(2), 249-254 (2000)

Publisher's Note Springer Nature remains neutral with regard to jurisdictional claims in published maps and institutional affiliations. 